\title{
MDR-TB and XDR-TB: drug resistance and treatment
}

\section{outcomes}

\section{To the Editors:}

We read with interest the study by HwANG et al. [1], which continues the discussion on factors associated with poor outcomes in the treatment of multidrug-resistant tuberculosis (MDRTB) and extensively drug-resistant tuberculosis (XDR-TB).

The subject is quite topical, as TB programmes and clinicians are searching for solutions to treat MDR/XDR-TB most effectively. Recently published studies and a systematic review have shown that XDR-TB is associated with higher probability of failure and death, and lower probability of treatment success than MDR-TB [2-7]. The XDR-TB-defining drugs have been studied to evaluate their role in determining successful outcomes in MDR/XDR-TB cases (XDR-TB is defined as resistance to at least isoniazid and rifampicin (MDR-TB) plus to any fluoroquinolone and at least one of three injectable antiTB drugs (amikacin, capreomycin or kanamycin) [2, 3]).

HWANG et al. [1] re-analysed data from previously published studies, including 197 MDR/XDR-TB cases in HIV-negative individuals (42 of them affected by XDR-TB) who were enrolled in a university hospital in Seoul, South Korea. They found that resistance to additional first-line anti-TB drugs (other than isoniazid and rifampicin) or to injectable drugs was not associated with higher odds for poor treatment outcomes in patients with MDR-TB. However, streptomycin resistance was associated with poor outcomes in XDR-TB cases, with high OR (12.05, 95\% CI 1.48-98.38). HwANG et al. [1] were not able to confirm our own previous findings [4], which reported a favourable outcome for cases with MDR-TB strains sensitive to kanamycin or capreomycin. HWANG et al. [1] call for larger studies to evaluate the role of injectables in MDR/XDR-TB treatment.

Recently, different classes of XDR-TB-defining drugs have been studied systematically by TBNET to evaluate their role in determining successful outcomes in MDR/XDR-TB cases. After demonstrating that XDR-TB patients have a higher risk of death and failure than those with MDR-TB [5-7], we found evidence that: 1) resistance to additional first-line drugs (other than isoniazid and rifampicin) is a predictor of adverse outcomes [7]; and 2) resistance to fluoroquinolones contributes to increased risk of death and failure in MDR-TB cases [8]. Additionally, the role of second-line injectable drugs was studied using logistic regression analysis; we found that resistance to capreomycin was the only independent variable significantly associated with unfavourable outcomes in MDR/ XDR-TB (OR 3.51, 95\% CI 1.67-7.36; $\mathrm{p}=0.001$ ), while resistance to amikacin (OR 1.76, 95\% CI 0.91-3.39; $\mathrm{p}=0.09$ ) and kanamycin (OR 1.57, 95\% CI 0.96-2.57; $p=0.07$ ) achieved borderline significance [8].

Following the report by HwANG et al. [1], we attempted to verify their findings using the TBNET cohort. The cohort consisted of 4,583 culture-confirmed cases diagnosed consecutively by the TB clinical reference centres in Estonia (Tallin and Tartu), Germany (Borstel, Grosshansdorf and Bad-Lippspringe), Italy (Sondalo, Milan and Rome) and the Russian Federation (Archangels Oblast). Amongst the cohort, 240 MDR-TB and 48 XDR-TB cases had a definitive outcome recorded (treatment success, died, failure). The standard definitions for treatment outcome in MDRTB were used [9]. Drug susceptibility testing (DST) for first-line (isoniazid, rifampicin, ethambutol and streptomycin) and second-line anti-TB drugs was performed according to World Health Organization (WHO) recommendations by qualityassured laboratories and retested at WHO's Supranational Reference Laboratories in Rome and Milan, Borstel, Stockholm (Sweden), and Oslo (Norway) [4, 8]. In all countries, regimens to treat MDR/XDR-TB cases were tailored to the DST results according to WHO recommendations. Within the cohort, the proportion of HIV-seropositive patients was $4.7 \%$ [4].

Data from all culture-confirmed MDR/XDR-TB cases with a definitive outcome recorded were re-analysed [4] to compare with the findings of HWANG et al. [1]. Logistic regression analysis was performed in order to evaluate the ORs associated with negative treatment outcomes (failure, death), comparing patients who were susceptible to additional first-line or injectable drugs versus those with different patterns of resistance to the above-mentioned drugs, respectively.

The results are reported in table 1 according to the format used in the study by HwANG et al. [1]. In contrast to their findings, we found no significant risk factor for negative treatment outcome using univariate analysis, even after adjusting for the main confounding variables. Streptomycin resistance was not associated with negative treatment outcomes in either the overall MDR/XDR-TB cohort (unadjusted and adjusted ORs were 1.07 with $95 \%$ CI $0.4-2.9$ and 1.13 with $95 \%$ CI $0.32-4.1$, respectively) or in XDR-TB cases specifically (unadjusted and adjusted ORs were 1.19 with $95 \%$ CI $0.1-20.2$ and 0.43 with 95\% CI 0.66-15, respectively).

MDR-TB and resistance to any fluoroquinolone plus one second-line injectable drug was in fact the only combination of drug resistance significantly associated with an increased risk of death or failure (unadjusted and adjusted ORs were 2.6, 95\% CI $1.38-4.88$ and $2.9,95 \%$ CI 1.51-5.63, respectively; table 1).

Our findings suggest that streptomycin resistance was not associated with death and failure in this European cohort. A number of factors may have contributed to the differences reported. Methodological differences (e.g. number of drugs tested for resistance, sample sizes) and data limitations of both retrospective studies make a clear explanation for the different outcomes of the analyses difficult to discern. From a clinical perspective, the mechanism whereby streptomycin resistance alone would produce poorer treatment outcomes is unclear and warrants additional exploration. 


\begin{tabular}{|c|c|c|c|c|c|}
\hline Groups & $\begin{array}{l}\text { Treatment } \\
\text { success }\end{array}$ & $\begin{array}{c}\text { Adverse treatment } \\
\text { outcome }\end{array}$ & Total & $\begin{array}{l}\text { Unadjusted OR } \\
\qquad(95 \% \mathrm{Cl})\end{array}$ & $\begin{array}{l}\text { Adjusted OR }{ }^{\#} \\
\qquad(95 \% \mathrm{Cl})\end{array}$ \\
\hline \multicolumn{6}{|l|}{ MDR-TB } \\
\hline Susceptible to all first-line & $4(57.1)$ & $3(42.9)$ & 7 & 1 & 1 \\
\hline Resistant to two first-line $^{+}$ & $123(65.8)$ & $64(34.2)$ & 187 & $0.69(0.15-3.19)$ & $1.63(0.13-21.31)$ \\
\hline Resistant to one second-line + any fluoroquinolone & $22(45.8)$ & $26(54.2)$ & 48 & $2.6(1.38-4.88)$ & $2.9(1.51-5.63)$ \\
\hline Susceptible to all second-line & $64(82.1)$ & $14(17.9)$ & 78 & 1 & 1 \\
\hline Resistant to one second-line & $53(74.7)$ & $18(25.3)$ & 71 & $1.55(0.71-3.41)$ & $1.31(0.52-3.29)$ \\
\hline Resistant to two or all second-line & $13(72.2)$ & $5(27.8)$ & 18 & $1.76(0.54-5.74)$ & $1.85(0.54-6.39)$ \\
\hline \multicolumn{6}{|l|}{ XDR-TB } \\
\hline Resistant to all second-line & $3(37.5)$ & $5(62.5)$ & 8 & $1.81(0.35-9.24)$ & $0.94(0.14-6.26)$ \\
\hline
\end{tabular}

Although results from the various published studies on MDR/ XDR-TB treatment outcomes do not leave us with a clear clinical direction to follow, the underlying messages are the same: MDR/XDR-TB is difficult to decode with the limited information available based on retrospective data from small cohorts [2, 10]. Well-designed, randomised, prospective studies are necessary to provide more definitive answers to the treatment questions that are still pending, the most burning being which regimens can offer MDR/XDR-TB patients the best chance of cure [2].

\section{G.B. Migliori*, G. Sotgiu", M. D’Arcy Richardson", R. Centis*, A. Facchini* , G. Guenther ${ }^{+}$, A. Spanevello ${ }^{\S}$ and C. Lange ${ }^{+}$for TBNET}

*WHO Collaborating Centre for TB and Lung Diseases, Fondazione S. Maugeri, Care and Research Institute, Tradate, \#Hygiene and Preventive Medicine Institute, University of Sassari, Sassari, `́Università degli Studi dell'Insubria, Varese, Italy. "HIV/TB Global Program, PATH, Seattle, Washington, WA, USA. ${ }^{+}$Division of Clinical Infectious Diseases, Medical Clinic, Research Centre Borstel, Borstel, Germany.

Correspondence: G.B. Migliori, WHO Collaborating Centre for TB and Lung Diseases, Fondazione S. Maugeri, Care and Research Institute/TBNET Secretariat (TuBerculosis Network European Trialsgroup)/Stop TB, via Roncaccio 16, 21049, Tradate, Italy. E-mail: giovannibattista.migliori@fsm.it

\section{REFERENCES}

1 Hwang SS, Kim HR, Kim HJ, et al. Impact of resistance to first-line and injectable drugs on treatment outcomes in MDR-TB. Eur Respir J 2009; 33: 581-585.

2 Sotgiu G, Ferrara G, Matteelli A, et al. Epidemiology and clinical management of XDR-TB: a systematic review by TBNET. Eur Respir J 2009; 33: 871-881.

3 Migliori GB, Loddenkemper R, Blasi F, et al. 125 years after Robert Koch's discovery of the tubercle bacillus: the new XDR-TB threat. Is "science" enough to tackle the epidemic? Eur Respir J 2007; 29: 423-427.

4 Migliori GB, Lange C, Centis R, et al. Resistance to second-line injectables and treatment outcomes in multidrug-resistant and extensively drug-resistant tuberculosis cases. Eur Respir J 2008; 31: 1155-1159.

5 Migliori GB, Ortmann J, Girardi E, et al. Extensively drug-resistant tuberculosis, Italy and Germany. Emerg Infect Dis 2007; 13: 780-782.

6 Migliori GB, Lange C, Girardi E, et al. Extensively drug-resistant tuberculosis is worse than multidrug-resistant tuberculosis: different methodology and settings, same results. Clin Infect Dis 2008; 46: 958-959.

7 Migliori GB, Besozzi G, Girardi E, et al. Clinical and operational value of the extensively drug-resistant tuberculosis definition. Eur Respir J 2007; 30: 623-626.

8 Migliori GB, Lange C, Girardi E, et al. Fluoroquinolones: are they essential to treat multidrug-resistant tuberculosis? Eur Respir J 2008; 31: 904-905.

9 Laserson K, Thorpe LE, Leimane V, et al. Speaking the same language: treatment outcome definitions for multidrug-resistant tuberculosis. Int J Tuberc Lung Dis 2005; 9: 640-645.

10 Migliori GB, Richardson MD, Lange C. Of blind men and elephants: making sense of extensively drug-resistant tuberculosis. Am J Respir Crit Care Med 2008; 178: 1000-1001. 